\title{
The fermi coupling, mass spectrum and decay properties of mesons within the covariant confined quark model
}

\author{
Gurjav Ganbold ${ }^{1,2, \star}$ \\ ${ }^{1}$ Bogoliubov Laboratory of Theoretical Physics, JINR, Dubna 141980, Russia \\ ${ }^{2}$ Institute of Physics and Technology, Ulaanbaatar 13330, Mongolia
}

\begin{abstract}
Main properties of stable quark-antiquark bound states have been investigated by using a relativistic quark model with analytic confinement. A new insight into the problem of hadron mass generation is provided. It has been shown that the compositeness condition expressing the Yukawa coupling of the meson-quark interaction and the master equation relating the meson mass function to the Fermi coupling can together guarantee the equivalency of the both theories, thereby providing an interpretation of the meson field as a bound state of constituent quarks. A smooth behavior for the Fermi coupling $G(M)$ obeying reasonable accuracy for the meson estimated masses $M$ has been obtained. We have also updated our previous numerical results for the weak-decay constants as well as the electromagnetic decay widths of mesons. The obtained estimates are found in reasonable agreement with the recent experimental data.
\end{abstract}

\section{Introduction}

Understanding of a number of phenomena in modern particle physics such as the quark confinement, hadronization, generation of hadron masses etc. requires correct description of hadron dynamics within theoretical models. Particularly, the Standard Model operates only fundamental particles (quarks, leptons, neutrinos), gauge and Higgs bosons, and it is not yet clear how to explain the appearance of the multitude of observed hadrons and elucidate the generation of their masses. The origin of the hadron masses is one of the puzzles of particle physics and the calculation of their mass spectra in a quality comparable to precise experimental data still remains a key problem in QCD.

Even before QCD was set up as the fundamental theory of strong interactions, it was understood that it is a difficult problem to describe a composite particle within QFT as based on the relativistic $S$-matrix. The original Lagrangian describes free fields and their interactions while the consideration of physical processes requires the renormalization, i.e. the transition from bare (unrenormalized) quantities to the physical (renormalized) ones. In particular, the bare field is related to the dressed one as $\phi_{0}=Z^{1 / 2} \phi_{r}$ through the wave function renormalization constant $Z$. The bare field $\phi_{0}$ may be eliminated from the Lagrangian by putting $Z=0$, that is the compositeness condition (CC). The CC requirement $Z_{H}=0$ is also one of the key ingredients in the covariant confined quark model (CCQM) development [1].

\footnotetext{
^e-mail: ganbold@ theor.jinr.ru
} 
Relativistic models with specific forms of analytically confined propagators have been developed to study some aspects of low-energy hadron physics [2]. Hereby, the role of analytic confinement in the formation of quark-antiquark bound states has been analyzed within Yukawa-type models. Additionally, the lowest mass of two-gluon bound state (the glueball) has been predicted in reasonable agreement with lattice estimations. A phenomenological model with an infrared confinement has been developed to study the dependence of the effective strong coupling $\alpha_{s}$ on the mass scale.

Below we apply the CCQM to the meson mass problem [3]. We use the CC requirement to determine the renormalized Yukawa coupling $g_{r}$ as a function of model parameters and observed hadron masses $m_{H}$. Our constraint master equation expresses the Fermi coupling $G$ as inversely proportional to the meson mass (self-energy) function that allows us to estimate the dependence $G\left(m_{H}\right)$ in a quite large region ranging from the $\pi$ up to $\Upsilon$ meson. We also compared the obtained dependence of $G\left(m_{H}\right)$ with the $\alpha_{s}$ coupling [2] and found their nontrivial behaviors at large mass region.

\section{Compositeness Condition and Mass Equation}

In quantum field theory description of hadrons as composite particles was thoroughly studied (e.g., in papers [4]). It may be shown (on an example of mesons) that bound state structure of hadrons can be described equivalently by using phenomenological Lagrangians of either Yukawa-type:

$$
\mathcal{L}_{\text {int }}^{Y}(x)=g_{H} H(x) J_{H}(x)
$$

or Fermi-type:

$$
\mathcal{L}_{\text {int }}^{F}(x)=\frac{G^{2}}{2} J_{H}^{2}(x),
$$

where $H(x)$ is a hadron field, $J_{H}(x)=\bar{q}(x) \Gamma_{H} q(x)$ is an interpolating current composed of constituent quarks, $g_{H}$ and $G$ are the renormalized Yukawa and Fermi couplings, respectively, $\Gamma_{H}$ is a spin matrix. For such equivalence one should fulfill two conditions: the first one is the $\mathrm{CC}$, which means that the renormalization constant of hadron field $Z_{H}$ is equal to zero

$$
Z_{H}=1-g_{H}^{2} \tilde{\Pi}_{H}^{\prime}\left(m_{H}^{2}\right)=0
$$

and the second one requires that the Fermi coupling $G$ should obey the normalization condition

$$
G \tilde{\Pi}\left(m_{H}^{2}\right)=1,
$$

where $\tilde{\Pi}_{H}\left(m_{H}^{2}\right)$ is the mass function of the hadron with mass $m_{H}$ and $\tilde{\Pi}_{H}^{\prime}(z)=d \tilde{\Pi}_{H}(z) / d z$.

Thus the vanishing of the wave function renormalization constant in the Yukawa theory may be interpreted as the elimination of any constituent bare field in the composite boson, while (4) determines the Fermi coupling.

The ideas proposed in [4] have been further pushed forward and successfully applied for study of different aspects of low-energy hadron physics: study of QCD bosonization and QCD vacuum, quark confinement and hadron phenomenology (form factors and decay properties of mesons, baryons and exotic states) [5]. We have recently proposed the CCQM model, where infrared quark confinement was introduced to guarantee the absence of all possible thresholds corresponding to quark production. The implementation of quark confinement allowed to use the same values for the constituent quark masses for the simplest quark-antiquark systems (mesons) and more complicated multiquark configurations (baryons, tetraquarks, etc.). It should be emphasized that the experimental values of hadron masses are used in all calculations performed in the CCQM. Up to now we have used only the compositeness condition in (3) to determine the renormalized Yukawa coupling $g_{H}$ as a function of meson mass and model parameters. In paper [3] we used the normalization condition (4) to investigate the dependence of the coupling $G$ on physical meson masses. 


\section{Meson Mass Function}

The interaction of the ground-state (pseudoscalar and vector) mesons with their constituent quarks is described in the CCQM by a Lagrangian:

$$
\mathcal{L}_{\text {int }}=g_{H} H(x) J_{H}(x), \quad J_{H}(x)=\int d x_{1} \int d x_{2} F_{H}\left(x ; x_{1}, x_{2}\right) \bar{q}_{2}\left(x_{2}\right) \Gamma_{H} q_{1}\left(x_{1}\right) .
$$

Here, $\Gamma_{P}=i \gamma^{5}$ and $\Gamma_{V}^{\mu}=\gamma^{\mu}$ for the pseudoscalar and vector mesons, respectively.

For the vertex function $F_{H}$ we use the translational invariant form

$$
F_{H}\left(x, x_{1}, x_{2}\right)=\delta\left(x-w_{1} x_{1}-w_{2} x_{2}\right) \Phi_{H}\left(\left(x_{1}-x_{2}\right)^{2}\right),
$$

where $w_{i}=m_{q_{i}} /\left(m_{q_{1}}+m_{q_{2}}\right)$. Its Fourier transform is chosen in a Gaussian form

$$
\tilde{\Phi}_{H}\left(-p^{2}\right)=\int d x e^{i p x} \Phi_{H}\left(x^{2}\right)=e^{p^{2} / \Lambda_{H}^{2}}
$$

The size parameter $\Lambda_{H}$ is an adjustable quantity. Since the calculation of the Feynman diagrams proceeds in the Euclidean region, where $p^{2}=-p_{E}^{2}$, the vertex function decreases rapidly for $p_{E}^{2} \rightarrow \infty$ and it provides ultraviolet convergence in the evaluation of any diagram.

By using (7) and the quark propagator in the Schwinger representation

$$
S_{q}(x-y)=\int \frac{d^{4} k}{(2 \pi)^{4} i} \frac{e^{-i k(x-y)}}{m_{q}-k}
$$

we finally rewrite the Fourier transform of the mass function as follows:

$$
\begin{aligned}
\tilde{\Pi}_{H}\left(p^{2}\right) & =\frac{3}{4 \pi^{2}} \int_{0}^{\infty} \frac{d t t}{a_{H}^{2}} \int_{0}^{1} d s e^{-t z_{0}+z_{H}}\left\{\frac{n_{H}}{a_{H}}+m_{q_{1}} m_{q_{2}}+\left(w_{1}-\frac{b}{a_{H}}\right)\left(w_{2}+\frac{b}{a_{H}}\right) p^{2}\right\}, \\
z_{0} & =s m_{q_{1}}^{2}+(1-s) m_{q_{2}}^{2}-s(1-s) p^{2}, \quad z_{H}=\frac{2 s_{H} t}{2 s_{H}+t}\left(s-w_{2}\right)^{2} p^{2}, \\
a_{H} & =2 s_{H}+t, \quad b=\left(s-w_{2}\right) t .
\end{aligned}
$$

Here $n_{P}=2, n_{V}=1$ and $s_{H}=1 / \Lambda_{H}^{2}$. Note, a branching point appears in (9) at $p^{2}=\left(m_{q_{1}}+m_{q_{2}}\right)^{2}$. At this point the integral over $t$ becomes divergent as $t \rightarrow \infty$ because of $z_{0}=0$ at $s=m_{q_{2}} /\left(m_{q_{1}}+m_{q_{2}}\right)$. However, one can avoid the appearance of the threshold singularity by introducing an infrared cutoff $\tilde{\Pi}_{H}\left(p^{2}\right)=\int_{0}^{\infty} d t \ldots \rightarrow \tilde{\Pi}_{H}\left(p^{2}, \lambda\right)=\int_{0}^{1 / \lambda^{2}} d t \ldots$ on the upper limit of the scale integration in (9). Hereby, the infrared cutoff parameter $\lambda$ has a dimension of energy (mass).

Now we can investigate the dependence of the Fermi coupling $G$ on the hadron masses, or estimate the mass by using (4) because the mass function $\tilde{\Pi}_{H}\left(p^{2}, \lambda\right)$ is well defined for finite $\lambda>0$.

\section{Meson Spectrum, Decay Constants and Widths}

A given meson $H$ is characterized by the coupling $g_{H}$, size parameter $\Lambda_{H}$ (characterizes the distribution of the quarks inside the hadron), and two of the constituent quark masses $m_{q}\left(m_{u}=m_{d}, m_{s}, m_{c}\right.$, $\left.m_{b}\right)$. The infrared confinement parameter $\lambda$ is universal for all hadrons.

\begin{tabular}{|c|c|c|c|c|c|}
\hline Parameters & $m_{u / d}$ & $m_{s}$ & $m_{c}$ & $m_{b}$ & $\lambda$ \\
\hline Values (GeV) & 0.235 & 0.442 & 1.61 & 5.07 & 0.181 \\
\hline
\end{tabular}


Table 1. The fitted values of the size parameters $\Lambda_{H}$ (in $\mathrm{GeV}$ ) [3].

\begin{tabular}{|c|c|c|c|c|c|c|c|c|c|}
\hline$\pi$ & $K$ & $D$ & $D_{s}$ & $B$ & $B_{s}$ & $B_{c}$ & $\eta_{c}$ & $\eta_{b}$ & \\
\hline 0.87 & 1.02 & 1.71 & 1.81 & 1.90 & 1.94 & 2.50 & 2.06 & 2.95 & \\
\hline \hline$\rho$ & $\omega$ & $\phi$ & $J / \Psi$ & $K^{*}$ & $D^{*}$ & $D_{s}^{*}$ & $B^{*}$ & $B_{s}^{*}$ & $\Upsilon$ \\
\hline 0.61 & 0.50 & 0.91 & 1.93 & 0.75 & 1.51 & 1.71 & 1.76 & 1.71 & 2.96 \\
\hline
\end{tabular}

The model parameters have been determined by fitting meson leptonic decay constants and electromagnetic decay widths, known either from experiment or, from lattice simulations. The results of the fit for the model parameters are given in (10) and in table 1, respectively.

The obtained results of our (overconstrained) least-squares fit [3] are displayed in tables 2 and 3.

Table 2. Fit values for the leptonic decay constants $f_{H}$ (in MeV) compared with experimental data.

\begin{tabular}{|l|c|l|l||l|l|l|l|}
\hline & Fit Values & Data & Ref. & & Fit Values & Data & Ref. \\
\hline$f_{\pi}$ & 128.4 & $130.4 \pm 0.2$ & {$[6,7]$} & $f_{\rho}$ & 221.2 & $221 \pm 1$ & {$[6]$} \\
$f_{K}$ & 156.0 & $156.1 \pm 0.8$ & {$[6,7]$} & $f_{\omega}$ & 204.2 & $198 \pm 2$ & {$[6]$} \\
$f_{D}$ & 206.7 & $206.7 \pm 8.9$ & {$[6,7]$} & $f_{\phi}$ & 228.2 & $227 \pm 2$ & {$[6]$} \\
$f_{D_{s}}$ & 257.5 & $257.5 \pm 6.1$ & {$[6,7]$} & $f_{J / \Psi}$ & 415.0 & $415 \pm 7$ & {$[6]$} \\
$f_{B}$ & 189.7 & $192.8 \pm 9.9$ & {$[8]$} & $f_{K^{*}}$ & 215.0 & $217 \pm 7$ & {$[6]$} \\
$f_{B_{s}}$ & 235.3 & $238.8 \pm 9.5$ & {$[8]$} & $f_{D^{*}}$ & 223.0 & $245 \pm 20$ & {$[10]$} \\
$f_{\eta_{c}}$ & 386.6 & $438 \pm 8$ & {$[9]$} & $f_{D_{s}^{*}}$ & 272.0 & $272 \pm 26$ & {$[10]$} \\
$f_{B_{c}}$ & 445.6 & $489 \pm 5$ & {$[9]$} & $f_{B^{*}}$ & 196.0 & $196 \pm 44$ & {$[10]$} \\
$f_{\eta_{b}}$ & 609.1 & $801 \pm 9$ & {$[9]$} & $f_{B_{s}^{*}}$ & 229.0 & $229 \pm 46$ & {$[10]$} \\
& & & & $f_{\Upsilon}$ & 661.3 & $715 \pm 5$ & {$[6]$} \\
\hline
\end{tabular}

Note, we did not include decay results for the $\eta\left(\eta^{\prime}\right)$-mesons because they have some additional features like the mixing angle and a possible significant gluon admixture to the conventional $q \bar{q}$ structure of the $\eta^{\prime}$. The constituent quark masses and the values for the size parameters fall into

Table 3. Fit values for basic electromagnetic decay widths (in keV) compared with PDG-2012 data.

\begin{tabular}{|l|c|c||l|c|c|}
\hline Process & Fit Values & Data [6] & Process & Fit Values & Data [6] \\
\hline$\pi^{0} \rightarrow \gamma \gamma$ & $5.07 \times 10^{-3}$ & $(7.7 \pm 0.4) \times 10^{-3}$ & $K^{* \pm} \rightarrow K^{ \pm} \gamma$ & 57.7 & $50 \pm 5$ \\
$\eta_{c} \rightarrow \gamma \gamma$ & 3.47 & $5.0 \pm 0.4$ & $K^{* 0} \rightarrow K^{0} \gamma$ & 129 & $116 \pm 10$ \\
$\rho^{ \pm} \rightarrow \pi^{ \pm} \gamma$ & 76.3 & $67 \pm 7$ & $D^{* \pm} \rightarrow D^{ \pm} \gamma$ & 0.59 & $1.5 \pm 0.5$ \\
$\omega \rightarrow \pi^{0} \gamma$ & 687 & $703 \pm 25$ & $J / \Psi \rightarrow \eta_{c} \gamma$ & 1.90 & $1.58 \pm 0.37$ \\
\hline
\end{tabular}

the expected range. The size parameters show the expected general pattern - the geometrical size of a meson, which is inversely proportional to $\Lambda_{H_{i}}$, decreases as the mass increases. The present numerical least-squares fit and the values for the model parameters supersede the results of our earlier analysis given in [1], where a different set of electromagnetic decays has been used. In the present fit we have also updated some of the theoretical/experimental input values.

Our prime goal is to study the behavior of the Fermi coupling $G$ in (4) as a function of the hadron masses by keeping other parameters (infrared cutoff parameter $\lambda$, size parameters $\Lambda_{H}$ and constituent quark masses $m_{q}$ ) fixed. The original dependence of $G$ on the hadron mass is obtained by directly taking the physical values, and results in a decreasing but sawtooth-like behavior. 
A smoothness criterion might be considered as a possibility, when values for the meson masses are computed through (4) as a function of the other model parameters. In table 4 we present our results for the dimensionless coupling $G \lambda^{2}$ in the case of exact fit (when the values of meson masses are taken from experimental data) and in the case of the smooth fit. The model meson masses estimated

Table 4. Estimations for dimensionless Fermi coupling $G \lambda^{2}$ in cases of exact and smooth fit [3].

\begin{tabular}{|c|c|c||c|c|c|}
\hline & Exact fit & Smooth fit & & Exact fit & Smooth fit \\
\hline$\pi$ & 1.508 & 1.507 & $\eta_{c}$ & 0.128 & 0.141 \\
$K$ & 0.919 & 0.920 & $J / \Psi$ & 0.129 & 0.139 \\
$\rho$ & 0.571 & 0.560 & $B$ & 0.215 & 0.125 \\
$\omega$ & 0.673 & 0.553 & $B^{*}$ & 0.237 & 0.124 \\
$K^{*}$ & 0.476 & 0.472 & $B_{s}$ & 0.192 & 0.122 \\
$\phi$ & 0.377 & 0.400 & $B_{s}^{*}$ & 0.232 & 0.121 \\
$D$ & 0.224 & 0.195 & $B_{c}$ & 0.0905 & 0.118 \\
$D_{s}$ & 0.197 & 0.184 & $\eta_{b}$ & 0.0612 & 0.0986 \\
$D^{*}$ & 0.168 & 0.180 & $\Upsilon$ & 0.0600 & 0.0984 \\
$D_{s}^{*}$ & 0.158 & 0.170 & & & \\
\hline
\end{tabular}

by using the smooth fit of $G$ are shown in table 5 .

Table 5. The fitted values for the meson masses (in MeV) [3].

\begin{tabular}{|c|c|c|c|c|c|}
\hline & Model & Data [6] & & Model & Data [6] \\
\hline$m_{\pi}$ & 141.0 & $139.57018 \pm 0.0003$ & $m_{\eta_{c}}$ & 2922.0 & $2983.7 \pm 0.7$ \\
$m_{K}$ & 493.0 & $493.677 \pm 0.016$ & $m_{J / \Psi}$ & 3067.0 & $3096.916 \pm 0.011$ \\
$m_{\rho}$ & 778.0 & $775.26 \pm 0.25$ & $m_{B}$ & 5425.0 & $5279.26 \pm 0.17$ \\
$m_{\omega}$ & 806.0 & $782.65 \pm 0.12$ & $m_{B^{*}}$ & 5450.0 & $5325.2 \pm 0.4$ \\
$m_{K^{*}}$ & 893.0 & $891.66 \pm 0.26$ & $m_{B_{s}}$ & 5524.0 & $5366.77 \pm 0.24$ \\
$m_{\phi}$ & 1011.0 & $1019.45 \pm 0.02$ & $m_{B_{s}^{*}}$ & 5566.0 & $5415.8 \pm 1.5$ \\
$m_{D}$ & 1915.0 & $1869.62 \pm 0.15$ & $m_{B_{c}}$ & 6041.0 & $6274.5 \pm 1.8$ \\
$m_{D_{s}}$ & 1998.0 & $1968.50 \pm 0.32$ & $m_{\eta_{b}}$ & 8806.0 & $9398.0 \pm 3.2$ \\
$m_{D^{*}}$ & 2001.0 & $2010.29 \pm 0.13$ & $m_{\Upsilon}$ & 8880.0 & $9460.30 \pm 0.26$ \\
$m_{D_{s}^{*}}$ & 2099.0 & $2112.3 \pm 0.5$ & & & \\
\hline
\end{tabular}

\section{Comparison of Fermi Coupling with Effective Strong Coupling}

It might be interesting to compare the behavior of smooth $G$ obtained in table 4 with the effective strong coupling $\alpha_{s}$ obtained in the QCD-inspired relativistic models with specific forms of analytically confined quark and gluon propagators [2]. In these models the nonlocal four-quark interaction is induced by one-gluon exchange between biquark currents, so, the resulting coupling $\alpha_{s}$ is dimensionless. The idea of such a comparison by re-scaling of one coupling to another, where both have a 'plateau' behavior, looks quite reasonable. Note that this plateau region is almost the same in both approaches. In figure 1 we compare the mass dependence of the re-scaled dimensionless Fermi coupling $\sim 1.74 G \lambda^{2}$ (solid line) in [3] with the effective strong coupling $\alpha_{s}$ (dashed line) in [2]. One can see that these two curves are different in the region of small mass because of different confinement mechanisms realized in original models. However, these curves behave very similar to each other in 
the intermediate and heavy mass regions above $\sim 2 \mathrm{GeV}$, that is a nontrivial observation and deserves additional consideration.

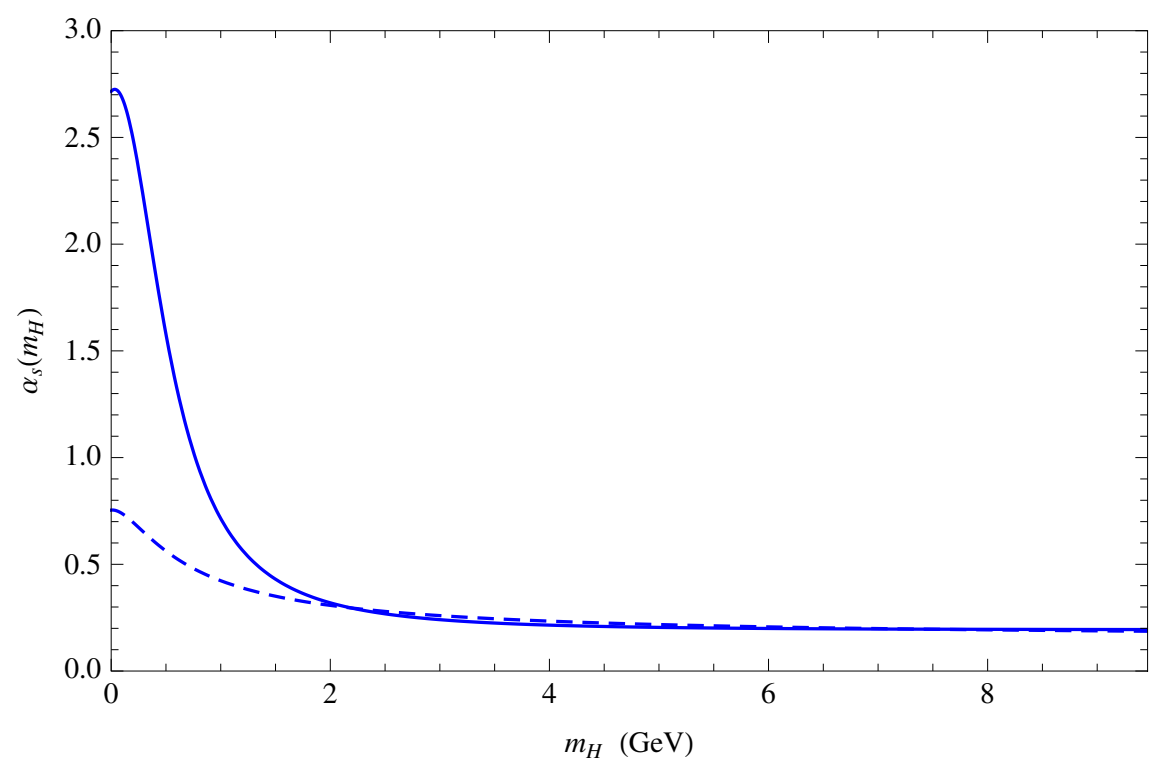

Figure 1. The dimensionless Fermi coupling $\lambda^{2} G\left(m_{H}\right)$ (solid line) scaled in the region of large masses to the effective strong coupling $\alpha_{s}\left(m_{H}\right)$ obtained in [2] (dashed line).

\section{References}

[1] T. Branz et al., Phys. Rev. D 81, 034010 (2010). M. A. Ivanov et al., Phys. Rev. D 85, 034004 (2012)

[2] G. Ganbold, Phys. Rev. D 79, 034034 (2009). Phys. Rev. D 81, 094008 (2010). Phys. Part. Nucl. 43, 79 (2012). Phys. Part. Nucl. 45, 10 (2014)

[3] G. Ganbold, T. Gutsche, M. A. Ivanov, V. E. Lyubovitskij, J. Phys. G 42, 075002 (2015)

[4] B. Jouvet, Nuovo Cim. 3, 1133 (1956). A. Salam, Nuovo Cim. 25, 224 (1962). M. A. Braun, Nucl. Phys. B 14, 413 (1969). S. Weinberg, Phys. Rev. 130, 776 (1963)

[5] G. V. Efimov and M. A. Ivanov, Int. J. Mod. Phys. A 4, 2031 (1989). M. A. Ivanov et al., Phys. Rev. D 56, 348 (1997). A. Faessler et al., Eur. Phys. J. C 4, 18 (2002). G. V. Efimov and G. Ganbold, Phys. Rev. D 65, 054012 (2002)

[6] J. Beringer et al. [Particle Data Group Collaboration], Phys. Rev. D 86, 010001 (2012)

[7] J. L. Rosner, S. Stone, arXiv:1002.1655 [hep-ex]

[8] J. Laiho, E. Lunghi, R. S. Van de Water, Phys. Rev. D 81, 034503 (2010)

[9] T. -W. Chiu et al. [TWQCD Collaboration], Phys. Lett. B 651, 171 (2007)

[10] D. Becirevic et al., Phys. Rev. D 60, 074501 (1999) 